\title{
Interatomic Coulombic Decay in mixed NeKr clusters
}

Tiberiu Arion ${ }^{1}$, Melanie Mucke ${ }^{1}$, Marko Förstel ${ }^{1,2}$, Alex M. Bradshaw ${ }^{1,3}$, Uwe Hergenhahn ${ }^{1}$ ${ }^{1}$ Max-Planck-Institut für Plasmaphysik, EURATOM Association, Boltzmannstr. 2, 85748 Garching bei München, Germany

${ }^{2}$ Max-Planck-Institut für Kernphysik, Saupfercheckweg 1, 69117 Heidelberg, Germany

${ }^{3}$ Fritz-Haber-Institut der Max-Planck-Gesellschaft, Faradayweg 4- 6, 14195 Berlin, Germany

Corresponding author: Uwe Hergenhahn, IPP, c/o Helmholtz-Zentrum Berlin, Albert-Einstein-Str. 15, 12489 Berlin, Germany.

Email: uwe.hergenhahn@ipp.mpg.de

\begin{abstract}
We report the occurrence of Interatomic Coulombic Decay (ICD) in mixed NeKr clusters. A well-defined feature ranging from $9-12 \mathrm{eV}$ in kinetic energy is observed in coincidence with the $\mathrm{Ne} 2 \mathrm{~s}$ photoelectrons. It derives from an ICD process, in which an initial $\mathrm{Ne} 2 \mathrm{~s}$ vacancy is filled by a $\mathrm{Ne} 2 \mathrm{p}$ electron and an electron is emitted from a $4 \mathrm{p}$ level on a neighboring $\mathrm{Kr}$ atom. We have studied the dependence of the effect on photon energy, cluster composition and cluster size. Interestingly, the ICD electron energy increases slightly and grows a shoulder on going from $2 \%$ to $5 \% \mathrm{Kr}$ in the co-expansion process, which we interpret in terms of surface vs. bulk effects.
\end{abstract}

Keywords:

ICD, Interatomic Coulombic Decay, Cluster, NeKr, Autoionization

PACS codes: 32.30.-r, 32.80.Fb, 32.80.Zb, 33.60.+q, 36.40.-c, 36.40.Mr 


\section{Introduction}

One of the best known examples of autoionization (a process in which an atom or molecule spontaneously emits an electron and thus undergoes a transition into a higher charge state) is Auger decay. ${ }^{1}$ Following inner shell ionization of the atom (or molecule), which leaves it in an excited state, a rearrangement occurs in order to reach minimum energy: An electron of lesser binding energy fills the inner-shell hole and a second electron from the same or another level is emitted. Auger decay is usually regarded as a process taking part in an individual atom or molecule. One may then ask the question as to whether this type of autoionization occurs in a different way when the atom or molecule is placed in a particular environment, e.g. in the solid or in a cluster. In the last decade it has been found that new autoionization channels can indeed open up in such a case, leading to final states with one vacancy located at the site of original ionization and the other on a neighboring atom or molecule. ${ }^{2-8}$ Typically, such a transition occurs at lower energy than most Auger processes and starts out from the ionization of an inner valence level. Both final state vacancies are located in outer valence levels. It is referred to as Interatomic (or Intermolecular) Coulombic Decay (ICD); more recently, a related process, termed Electron Transfer Mediated Decay (ETMD) has also been discovered..$^{9,10}$

In order for ICD to take place, a "minimum energy criterion" has to be fulfilled, namely, the binding energy of the inner valence electron, the emission of which starts the process, must exceed the double ionization potential (DIP) of the system. For the final states in question, the relevant limit is the sum of the binding energies of both outer valence electrons taking part in the decay, to which the Coulomb repulsion energy of the two vacancies in the final state needs to be added..$^{27,8,11,12}$ Therefore, the total energy of the final state in the case of ICD can be lower than in Auger decay, because the Coulomb energy of the distributed vacancies in the ICD case is lower than the one of a localized two-hole state in the Auger case. Singly ionized 
states which cannot decay by Auger emission can be unstable against ICD. Experimentally and theoretically it has been found that ICD takes place on a time-scale of the order of 1-100 fs. ${ }^{13-16}$ Therefore, in weakly bound systems the decay via ICD is more efficient than radiative decay paths or relaxation of the system involving nuclear dynamics. ${ }^{2-4,8}$ Although ICD is expected to be a universally occurring phenomenon, ${ }^{2,8,14}$ ICD-related experiments have so far been performed mainly on rare gas clusters and water clusters. Due to the fact that the photoemission spectrum from a solid is characterized by a strong background of "true secondary" electrons at very low kinetic energy and that coincidence experiments in this region are hence difficult to perform, clusters have been the system of choice. They not only resemble solids in structure and electronic behavior (see, e.g. Refs. 17-19), but are also easy to prepare. Our study on mixed rare gas clusters has two motivations: 1 . They are suitable prototypes to investigate whether ICD can be used in research on the structure of weakly bonded systems ${ }^{20}$. 2. Simple estimates for ICD in Ne-Kr give an exceptionally large transition energy of around $10 \mathrm{eV}$, resulting in an isolated spectral feature. This also makes $\mathrm{Ne}-\mathrm{Kr}$ a very suitable system for more refined spectroscopic studies on ICD, e.g. in the time domain.

In the present article we report on ICD observed in mixed $\mathrm{Ne}-\mathrm{Kr}$ clusters, formed by coexpansion of a $\mathrm{Ne} / \mathrm{Kr}$ gas mixture into vacuum. We identify the ICD signal as coming from the mixed aggregates in the following process:

$$
\begin{aligned}
& \mathrm{Ne}_{\mathrm{N}} \mathrm{Kr}_{\mathrm{M}}+h \nu \rightarrow \mathrm{Ne}_{\mathrm{N}-1}\left(\mathrm{Ne}^{+} 2 \mathrm{~s}^{-1}\right) \mathrm{Kr}_{\mathrm{M}}+\mathrm{e}_{\mathrm{ph}}^{-} \\
& \rightarrow \mathrm{Ne}_{\mathrm{N}-1} \mathrm{Kr}_{\mathrm{M}-1}\left(\mathrm{Ne}^{+} 2 \mathrm{p}^{-1}\right)\left(\mathrm{Kr}^{+} 4 \mathrm{p}^{-1}\right)+\mathrm{e}_{\mathrm{ph}}^{-}+\mathrm{e}^{-}{ }_{\mathrm{ICD}}
\end{aligned}
$$

Furthermore, in our study we have systematically studied the variation in form and position of the ICD peak as a function of the composition of the gas mixture and of size of the cluster. In earlier work, an ICD signal from other mixed rare gas systems has been identified, namely, for larger Ne-Ar aggregates in Refs. 20-21, for the He-Ne dimer in Ref. 22 and as a second step after Auger decay in Ar-Kr dimers. ${ }^{23}$ ICD in mixed rare gas clusters has also been the 3 
subject of numerous theoretical studies, see e.g. Ref.s 24-26 for NeAr, Ref.s 27 and 15 for $\mathrm{MgNe}$ and Ref. 28 for $\mathrm{Ne}$ doped He droplets (large He clusters). An Interatomic Coulombic Decay with transition energies around $10 \mathrm{eV}$ has also been found in the He dimer. ${ }^{29,30}$ There, however, the ICD initial states are 1s-np photoelectron satellites, whereas we consider the decay after ionization into an inner valence main line, which - in comparison - receives a larger oscillator strength.

\section{Experimental setup}

The established method of producing rare-gas clusters is to expand the gas through a submillimeter nozzle, from a reservoir at a pressure of the order of 1-20 bar, into a vacuum chamber held at a pressure of the order of $10^{-3}-10^{-4}$ mbar. The supersonic jet thus obtained can then be directed through a skimmer with a diameter of the order of 50-500 $\mu \mathrm{m}$ before entering the experimental chamber. By this so-called supersonic expansion method, ${ }^{31}$ one can produce homogeneous as well as heterogeneous (mixed) gas clusters. Mixed rare-gas clusters can be obtained via two different procedures: 1. A rare-gas cluster jet formed from one species is passed through an atmosphere of the second species in which atoms of the second type adsorb on the already existing clusters. This procedure is called doping or pick-up. 2. The desired mixture of gases is pre-formed and expanded through the nozzle-skimmer system into the experimental chamber. This method is known as co-expansion. The ensuing cluster structure from the two methods is not necessarily identical (see Ref. 32). For pick-up of $\mathrm{Kr}$ atoms by large $(N>1000) \mathrm{Ne}$ clusters a core-shell structure with a $\mathrm{Kr}$ core surrounded by $\mathrm{Ne}$

outer layers has been found. ${ }^{33}$ In our experiments the $\mathrm{Ne}-\mathrm{Kr}$ mixed clusters were prepared by co-expansion. No earlier experiments using this method of preparation are known to the authors. For mixtures of $\mathrm{Ne}$ and Ar, however, several studies have arrived at a structure analogous to Ref. 33: A core of the species with the higher freezing temperature (Ar) is 
surrounded by few atoms or solid layers of the more volatile species $(\mathrm{Ne}) .^{20,21} \mathrm{We}$ therefore assume our clusters also have a $\mathrm{Kr}$ core/Ne shell structure.

The initial mixing ratio was varied in the experiments from $2 \%$ to $5 \% \mathrm{Kr}$ atoms in the $\mathrm{Ne}-\mathrm{Kr}$ mixture. The initial mixing ratio is not, however, identical with the proportions with which the two species are found in the cluster. The decisive factor influencing the composition of the final clusters are the van der Waals interactions between the atoms. These are reflected, for instance, in the dimer binding energies for the three possible combinations of bonds $(\mathrm{Ne}-\mathrm{Ne}$ dimer binding energy: $3.6 \mathrm{meV}, \mathrm{Kr}-\mathrm{Kr}$ dimer binding energy: $17.3 \mathrm{meV}, \mathrm{Ne}-\mathrm{Kr}$ dimer binding energy: $6.1 \mathrm{meV})^{34-36}$ It is clear that $\mathrm{Kr}$ atoms will condense more easily, and at higher nozzle temperatures than $\mathrm{Ne}$ atoms. The clusters formed in the co-expansion are therefore richer in $\mathrm{Kr}$ than the initial gas mixture, while the agglomeration of $\mathrm{Ne}$ atoms ( $\mathrm{Ne}$ clustering) is not favored. A quantitative study of this relation for the case of a Ne-Ar mixture can be found in Ref. 21.

The cluster source has been described previously. ${ }^{37,38}$ In short, the gas mixture is prepared in the external reservoir with the desired composition and then expanded into vacuum through a $100 \mu \mathrm{m}$ diameter nozzle with a half opening angle of $15^{\circ}$. The nozzle can be cooled down by using liquid $\mathrm{N}_{2}$ or liquid $\mathrm{He}$, depending on the desired working temperature. For temperature stabilization, the system is fitted with an electronically controlled heater. The central part of the supersonic gas jet is then led into the interaction chamber via a conical skimmer $(1 \mathrm{~mm}$ in diameter, Beam Dynamics, Inc., Jacksonville, USA), where it crosses the soft x-ray radiation in the interaction region of the electron spectrometer. Stagnation pressures and expansion temperatures in our experiments were typically 1.5 bar and $115 \mathrm{~K}$, and are detailed in the Supplementary Material. ${ }^{39}$

The experiments were performed on the TGM-4 beamline of the BESSY II synchrotron light source in Berlin, Germany, in single-bunch mode, with photon energies between 55 and 5 
$110 \mathrm{eV}$. The spectrometer is a newly built magnetic bottle-type ${ }^{40}$ electron energy analyser based on a design by Lablanquie, Eland et $a l^{41}$ adapted to the situation at BESSY; it is an improved version of the instrument previously used for the investigation of ICD in $\mathrm{H}_{2} \mathrm{O}$ clusters. ${ }^{5}$ This recently commissioned device has been described in detail by Mucke et al. ${ }^{42}$ and has been successfully used for photoelectron spectroscopy of Ar clusters ${ }^{18,19}$ and of a fast beam of anions $\left(\mathrm{OH}^{-}\right.$and $\left.\mathrm{O}^{-}\right)$produced by an optical laser. ${ }^{43,44}$ In short, the experimental setup makes use of an inhomogeneous magnetic field to extract and parallelize the electrons emerging from the ionization processes and a homogeneous magnetic field to guide the electrons to a microchannel plate (MCP) detector. In our set-up, the total length of the drift tube is at $60 \mathrm{~cm}$. The inhomogeneous field is produced by a permanent magnet of $200 \mathrm{mT}$ that is placed few mm away from the interaction region. In front of the magnet, a 117.6 lines-perinch copper mesh (Precision Eforming LLC, Cortland, USA) is electrically shielding the magnet from the interaction region. This allows a voltage to be applied on the magnet in order to hinder secondary electrons produced on its surface from penetrating the interaction region, but does not electrically influence the point of intersection between the cluster and synchrotron beams. The homogeneous magnetic guiding field is produced by a copper solenoid which is wrapped around a drift tube, outside the vacuum. The tube is separated from the interaction volume by an aperture, and is terminated by a copper mesh few mm in front of the MCP stack. The spectrometer is designed such that, if desired, a retarding potential (improved energy resolution) or an accelerating potential (improved collection angle) can be applied along the interaction region. For the experiments presented here we have applied a retarding potential of $U_{\mathrm{ap}}=\mathrm{U}_{\mathrm{dt}}=-2.5 \mathrm{~V}$ on the aperture and drift tube, and $\mathrm{U}_{\text {mesh }}=+2.5 \mathrm{~V}$ on the mesh. The magnet behind the mesh was kept at a constant voltage of $U_{\text {magnet }}=+10.0 \mathrm{~V}$.

Electron energies measured by a magnetic-bottle type spectrometer are inferred from the time-of-flight of the electrons, which are on the order of some hundred ns in our instrument. 
Use of the BESSY single bunch mode is therefore a prerequisite for application of this technique. Time-to-energy conversion in our experiment was performed from reference measurements of noble gas photoelectron lines. The comparatively short length of the drift tube, and the large spot-size at the TGM-4 beamline lead to an energy resolution of approximately $\mathrm{E} / \Delta \mathrm{E}=20$.

For an ionization process in which two electrons $\left(\mathrm{e}_{1}\right.$ and $\left.\mathrm{e}_{2}\right)$ are produced, both are captured and guided efficiently to the MCP detector due to the large solid angle of collection, ${ }^{5,40,41}$. Event-based data acquisition is accomplished by use of a multi-hit capable time-to-digital converter (TDC) with 60 ps bin width (GPTA, Berlin, Germany). The background, as estimated from a part of the spectrum in which only electron pairs produced by two different synchrotron radiation pulses occur (not shown here), was subtracted in the offline analysis. This background of random coincidences is, however, quantitatively not significant for the data shown here.

The advantages of using such an electron spectrometer are the large acceptance angle, almost $4 \pi \mathrm{sR}$, and the good transmission at low kinetic energies ${ }^{5,40,41}$, which is necessary for studying most ICD processes. ${ }^{2,3,8,24,45-47}$ These features, which both lead to relatively short accumulation times per spectrum (less than 30 minutes), make the combination of magnetic bottle spectrometer and coincidence technique a very useful and effective tool to investigate such autoionization processes.

\section{Results and discussion}

As mentioned earlier, in order for the ICD process to become possible, a minimum amount of energy needs to be transferred to the system. For the case of a $\mathrm{NeKr}$ mixture, mixed ICD starts with $\mathrm{Ne} 2$ s ionization corresponding to the emission of a $\mathrm{Ne} 2 \mathrm{~s}$ electron with an atomic 
electron binding energy of $\mathrm{E}_{\mathrm{b}}=48.5 \mathrm{eV} .^{48,49}$ The hole is then filled by a Ne $2 \mathrm{p}$ electron (atomic electron binding energies $E_{b} 1 / 2$, Ne atom $=21.7 \mathrm{eV}$ and $E_{b} 3 / 2$, Ne atom $=21.6 \mathrm{eV}$ ) (Ref. 49) and the remaining energy is transferred to a neighboring $\mathrm{Kr}$ atom, leading to the emission of a $4 \mathrm{p}$ electron $\left(\mathrm{E}_{\mathrm{b} 1 / 2, \mathrm{Kr} \text { atom }}=14.7 \mathrm{eV}\right.$ and $\left.\mathrm{E}_{\mathrm{b} 3 / 2, \mathrm{Kr} \text { atom }}=14.0 \mathrm{eV}\right) .{ }^{50}$ Allowing for some deviation of the energy levels from the atomic values due to cluster formation, the kinetic energy of the ICD electron will lie in the $[8 \mathrm{eV}, 12 \mathrm{eV}]$ interval. Taking the NeKr dimer as a concrete example the calculated value is $9.09 \mathrm{eV}$ for an experimentally determined average interatomic separation of $3.76 \AA$ (Ref. 51).

In Figure 1, the main panel (a) represents a coincident electron pair spectrum of $\mathrm{NeKr}$ clusters obtained from a mixture with $5 \% \mathrm{Kr}$ at a photon energy of $h v=110 \mathrm{eV}$. Pixels in the colorcoded map represent the number of events for which both electrons emitted in the ionization process have been recorded. The kinetic energy of the fast electron (denoted by $\mathrm{e}_{1}$ ), is displayed on the vertical axis, and the slower electron $\left(\mathrm{e}_{2}\right)$ on the horizontal axis. Regions of strong intensity in panel (a) of Fig. 1 identify the energies of electron pairs, which are emitted due to simultaneous or sequential photo-double-ionization. Several such regions are visible in the Fig., which arise due to different processes. Corresponding to the estimated ICD kinetic energy of 8-12 eV, plotted on the $\mathrm{e}_{2}$ axis, we find one distinct feature which arises at an $\mathrm{e}_{1}$ kinetic energy of approximately $61 \mathrm{eV}$. This $\mathrm{e}_{1}$ energy corresponds to a binding energy of $\sim 49$ $\mathrm{eV}$, which fits satisfactorily to an ionization from the Ne 2 s level in clusters. ${ }^{16}$ We therefore identify this feature in the map of two-electron processes with the sought after ICD process: The $\mathrm{Ne} 2 \mathrm{~s}^{-1}$ states in $\mathrm{NeKr}$ decay into mixed two-hole vacancy states according to the reaction eq. (1). Additional evidence follows. While the broadening of the feature along the $\mathrm{e}_{2}$ axis is mostly intrinsic, much of the broadening along the photoelectron energy axis $\left(\mathrm{e}_{1}\right.$ axis in Fig. 1) results from instrumental effects, and amounts to approx. $3 \mathrm{eV}$ at this kinetic energy. In the same manner as above, the most intense coincidence features that are seen for 
$\mathrm{e}_{2}$ kinetic energies of about 15.8 and $17 \mathrm{eV}$ can be identified with $\mathrm{Kr} 3 \mathrm{~d}$ photoelectrons (atomic electron binding energies ${ }^{50} 93.8$ and $95.0 \mathrm{eV}$, binding energies in $\langle N\rangle=1000$ clusters were found about $0.9 \mathrm{eV}$ lower ${ }^{52}$ ) in coincidence with $M N N$ Auger electrons. ${ }^{53,54}$ It should be pointed out that we always display the slower of the two released electrons along the $e_{2}$ axis. Depending on the binding energy of the vacancy that is initially produced, this will be either the photoelectron or the electron emitted by the autoionization process. The time-of-flight difference that distinguishes the two electrons in Fig. 1 occurs entirely in the analyser due to the difference in kinetic energy. Any intrinsic difference in the time of appearance of the two electrons would be in the fs range and is completely negligible in comparison. Only electron pairs with some difference in kinetic energy are plotted in the coincidence map. This is because the acquisition electronics have a dead time after having accepted an event. Some continuous features can also be distinguished in Fig. 1. The diagonal lines extending from upper left to lower right (emphasized by the green dashed lines), the most prominent one of them starting at $\left(\mathrm{e}_{1}, \mathrm{e}_{2}\right)$ kinetic energies of $(37.5 \mathrm{eV}, 6.5 \mathrm{eV})$, are attributed to direct photodouble-ionisation of uncondensed $\mathrm{Ne}$ into the $2 \mathrm{p}^{4}$ doubly ionized states, with binding energies between $\mathrm{E}_{\mathrm{b}}\left(2 \mathrm{p}^{4}\right)=62.5$ and $69.4 \mathrm{eV} .^{55}$ In this process an arbitrary energy sharing between the two electrons is possible, i.e. only their total kinetic energy is fixed. Therefore, two-electron events can appear anywhere on a diagonal line defined by $E_{k}\left(e_{1}\right)+E_{k}\left(e_{2}\right)=h v-E_{b}\left(2 p^{4}\right)$. The faint striations extending from lower left to upper right are due to an artefact of the acquisition electronics.

Summing the coincident events in the $e_{1}$ kinetic energy interval of [ $57 \mathrm{eV}, 64 \mathrm{eV}$ ] (marked by the red horizontal lines) along the $\mathrm{e}_{1}$ axis, for all values of $\mathrm{e}_{2}$, we obtain the spectrum shown in the upper-left panel of Fig. 1 (panel b)). In analogy to earlier experiments on rare-gas clusters ${ }^{3}$ and water clusters ${ }^{5}$, and following the discussion above, we interpret the broad feature in the $[8 \mathrm{eV}, 12 \mathrm{eV}] \mathrm{e}_{2}$ energy interval in the upper-left spectrum as the kinetic energy spectrum of 
Interatomic Coulombic Decay in NeKr mixed rare-gas clusters. Some intensity which is seen at higher $\mathrm{e}_{2}$ kinetic energies is due to $\mathrm{Kr} 3 \mathrm{~d}$ photoelectrons, which have some overlap with the selected summation interval when they are detected in coincidence with an $M N N$ Auger electron. By summing the coincident events along the $\mathrm{e}_{2}$ axis for all possible $\mathrm{e}_{1}$ kinetic energies, one obtains the spectrum of fast electrons shown in the panel on the right-hand side (panel c)). The structure between 35 and $60 \mathrm{eV}$ of kinetic energy (see notations in Fig $1 \mathrm{c}$ )) fits well to three groups of $\operatorname{Kr} M_{4,5} N N$ Auger lines discussed in earlier publications. ${ }^{53,54}$ The Ne 2 s photoline appears in this plot as a shoulder on the most energetic unresolved Auger line. Since we have derived Fig. 1 from the detected electron pairs only, electron lines due to conventional single photoionization are absent. The only process which can lead to the appearance of events related to $\mathrm{Ne} 2 \mathrm{~s}$ photoionization in the figure is the emission of a second electron due to ICD.

Similarly to Fig. 1, the main panel of Fig. 2 shows the $\left(e_{1}, e_{2}\right)$ coincident spectrum of $\mathrm{NeKr}$ mixed clusters $(3 \% \mathrm{Kr}$ in $\mathrm{NeKr}$ mixture) recorded at a photon energy of hv $=55 \mathrm{eV}$ as a color-coded map. For consistency with the spectra of Fig. 1, the kinetic energy of the faster electron $e_{1}$ is plotted on the vertical axis and the kinetic energy of the slower electron $e_{2}$ on the horizontal axis. However, as the kinetic energy of the photoelectron $(\sim 6.5 \mathrm{eV})$ is smaller than the expected kinetic energy of the ICD electron, the spectrum in the upper-left panel obtained by summing along the $e_{1}$ axis for all $e_{2}$ - shows in this case the photoelectron spectrum. It resembles the feature on the left-hand side in Figure 1, panel b. The spectrum in the right-hand panel then displays the ICD electron energy spectrum, obtained by summing along the $\mathrm{e}_{1}$ axis for all $\mathrm{e}_{2}$ energies. The broad background underneath the ICD feature is produced by the electrons emitted after $\mathrm{Kr} 4 \mathrm{p}$ ionization $\left(\mathrm{E}_{\mathrm{b}} 1 / 2, \mathrm{Kr}\right.$ atom $=14.7 \mathrm{eV}$ and $\mathrm{E}_{\mathrm{b}} 3 / 2, \mathrm{Kr}$ atom $=14.0 \mathrm{eV}) .{ }^{51} \mathrm{We}$ suggest that the $\mathrm{Kr} 4 \mathrm{p}$ electrons on their way out of the cluster ionize a Ne $2 p$ electron via collision. A part of the kinetic energy of the $4 p$ electron is used to 
overcome the $\mathrm{Ne} 2 \mathrm{p}$ binding energy and the rest can be divided between the two electrons in a continuous way. Similar to the case of direct photo-double-ionization discussed above the energy sharing between the two electrons released by photoionization + inelastic electron scattering is arbitrary, and the respective electron pairs will appear in the coincidence map as a broad diagonal feature of constant energy sum of the two electrons. One can see, comparing with Figure 1, that the ICD feature - in coincidence with the Ne 2 s photoelectrons - occurs in the same kinetic energy range $([8 \mathrm{eV}, 12 \mathrm{eV}])$, whereas that of the $\mathrm{Ne} 2 \mathrm{~s}$ photoelectrons depends on the photon energy. The invariance of the ICD electron kinetic energy for initial ionization at different photon energies is in agreement with the expected ICD behavior. Similar experiments have also been performed at $\mathrm{hv}=105 \mathrm{eV}$ (not shown here), in which the ICD electron-Ne 2 s photoelectron coincidence peak can be identified. However, as the $\mathrm{Kr} 3 \mathrm{~d}$ photoelectrons are now $5 \mathrm{eV}$ lower in kinetic energy they appear in the same energy range as the ICD electron. The two features from (Ne 2s, NeKr mixed ICD) coincidences and from (Kr $3 \mathrm{~d}, M_{4,5} N_{2,3} N_{2,3}$ Auger) coincidences ${ }^{53}$ cannot be completely separated.

Figures 3 (a) and (b) show a series of ICD spectra as recorded at $h v=55 \mathrm{eV}$ and $\mathrm{hv}=110 \mathrm{eV}$, respectively, for three different initial gas mixing ratios of $2 \% \mathrm{Kr}$ content (triangles), $3 \% \mathrm{Kr}$ (circles) and 5\% $\mathrm{Kr}$ (squares). All traces have been normalized to the same ICD peak height. Please note that, as already mentioned in the discussion of Fig. 2, in Fig. 3a the ICD spectrum sits on top of a background yielded after $\mathrm{Kr} 4 \mathrm{p}$ ionization, which is, due to energetic reasons, absent in Fig. 3b. The dashed vertical lines in both panels (a) and (b) indicate the center of gravity of the ICD peaks. One can observe that, on increasing the amount of $\mathrm{Kr}$ in the mixture, the ICD peak develops a shoulder on the high kinetic energy side and shifts slightly towards higher kinetic energies, from $10.4 \mathrm{eV}$ (for $2 \% \mathrm{Kr}$ ) to $10.7 \mathrm{eV}$ (for $3 \% \mathrm{Kr}$ ) and 11.1 $\mathrm{eV}$ (for $5 \% \mathrm{Kr}$ ), for $\mathrm{hv}=55 \mathrm{eV}$. For $\mathrm{hv}=110 \mathrm{eV}$ the same analysis yields $9.7 \mathrm{eV}$ (for $2 \% \mathrm{Kr}$ ), $9.8 \mathrm{eV}$ (for $3 \% \mathrm{Kr}$ ) and to $10.2 \mathrm{eV}$ (for $5 \% \mathrm{Kr}$ ). A possible explanation of the shift is that on 
enriching the mixture with $\mathrm{Kr}$, the mixed cluster size will increase. (In our expansion $\mathrm{Kr}$ condenses much more readily than Ne. We cannot quantify the degree of condensation here, but for analogous experiments on NeAr mixtures typically most of the Ar, but only a small fraction of $\mathrm{Ne}$ is found in clusters. Thus an increased $\mathrm{Kr}$ content immediately shows up in the cluster size.) Larger clusters are more polarizable than smaller clusters, which has the effect that both the initial and the final states are lowered in binding energy compared to smaller aggregates. However, as the final state is doubly ionized, the lowering of the binding energy by polarization screening will be larger than for the initial state. In effect, this will lead to a higher kinetic energy of the ejected ICD electrons. ${ }^{56}$

Based on the earlier results of Lundwall et al. for Ar-Ne and Ar-Kr mixed clusters, ${ }^{21,57}$ we can safely assume that in the mixed co-expanded $\mathrm{Ne}-\mathrm{Kr}$ clusters the $\mathrm{Kr}$ atoms will tend to concentrate in the core of the aggregates. For mixtures which are poor in $\mathrm{Kr}$, closed layers of $\mathrm{Ne}$ will form atop of a $\mathrm{Kr}$ core. Thus, for $\mathrm{Ne}$ at the surface, every $\mathrm{Ne}$ atom has $\mathrm{Ne}$ nearest neighbors as well as some that are $\mathrm{Kr}$. When the initial mixture is enriched with $\mathrm{Kr}$ the size of the $\mathrm{Kr}$ core increases and the layers of $\mathrm{Ne}$ start to get thinner. That is saying for a $\mathrm{Ne}$ atom at the $\mathrm{Ne}-\mathrm{Kr}$ interface (the only ones which can participate in mixed ICD) the number of $\mathrm{Kr}$ nearest neighbors increases. This allows us to interpret the main part of the ICD peak observed for both smaller and larger clusters in terms of Interatomic Coulombic Decay involving $\mathrm{Kr}$ and a $\mathrm{Ne}$ atom in a rather compact $\mathrm{Ne}$ layer at the surface. The shoulder appearing towards higher kinetic energies for larger cluster sizes (i.e., larger Kr cluster cores) will then be the signature of ICD electrons emitted from a process starting with a $2 \mathrm{~s}$ electron from a $\mathrm{Ne}$ atom at the cluster surface which is surrounded mostly by $\mathrm{Kr}$ atoms. Since $\mathrm{Kr}$ is more polarizable than $\mathrm{Ne}$, this will lead to a lowering in final state energy and thus to an increase in transition energy. 
This explanation assumes that the bond distance between the $\mathrm{Ne}$ and the $\mathrm{Kr}$ atom participating in mixed ICD does not change much on varying the overall cluster size. In principle, a change of ICD transition energy could equally well be caused by a change in separation of the $\mathrm{Ne}^{+}$ and the $\mathrm{Kr}^{+}$cation in the final state. We do not expect such changes because of differences in the initial state $\mathrm{Ne}-\mathrm{Kr}$ distance, since the interface of the $\mathrm{Kr}$ core and the $\mathrm{Ne}$ surface should be a local quantity which is rather independent of the size of the $\mathrm{Kr}$ core. Nor has an influence of the nuclear dynamics initiated by the photoionization process been seen in most inner valence dipole-allowed ICD processes. ${ }^{8}$ A full characterization of the mixed ICD process, including the shape of the outer valence band and the competition between $\mathrm{Ne}-\mathrm{Ne}$ and $\mathrm{Ne}-\mathrm{Kr} \mathrm{ICD}$, would be a worthwhile topic for future research though.

Taking into account that for a constant mixing ratio the size of the aggregates increases with both the increase of the stagnation pressure $\left(\mathrm{p}_{\mathrm{stag}}\right)$ and the decrease of the nozzle temperature $\left(\mathrm{T}_{\text {nozzle }}\right)$, we have also investigated ICD as a function of cluster size (not shown here). However, within the accuracy of the experiment changes in the ICD peak position as a function of the expansion conditions are not significant. This might be interpreted as evidence that the $\mathrm{NeKr}$ signal we receive is characteristic for the interface between the two rare gas layers only, and not for the cluster structure as a whole.

\section{Conclusions}

Mixed NeKr clusters produced by co-expansion of the two gases have been investigated using electron-electron coincidence spectroscopy. The cluster composition was varied by changing the $\mathrm{Kr}$ content of the initial gas mixture $(2 \% \mathrm{Kr}, 3 \% \mathrm{Kr}$ and $5 \% \mathrm{Kr})$. We have demonstrated the occurrence of Interatomic Coulombic Decay (ICD) in the decay of $\mathrm{Ne} 2 \mathrm{~s}^{-1}$ ionic states, similar to earlier findings in heterogeneous weakly bound rare gas clusters. ${ }^{20,21}$ The ICD feature has an unusually high kinetic energy of about $10 \mathrm{eV}$, thus separating it more clearly in 
energy from the secondary electrons and making the system a potential candidate for further spectroscopic characterization. Further, the results show that the ICD feature shifts towards higher kinetic energies, on increasing the amount of $\mathrm{Kr}$ in the gas mixture, but not on increasing the cluster size at the same mixing ratio. We have explained this by the change in the polarizability of the clusters as the cluster size increases due to $\mathrm{Kr}$ enrichment, thus leading to a lowering of the initial and the final states, with the latter being lowered more than the former. In the mixing ratio dependence it was observed that the cluster size increase due to $\mathrm{Kr}$ enrichment also produces a shoulder on the high kinetic energy side of the ICD feature. This is interpreted as evidence of the involvement of both surface and bulk $\mathrm{Kr}$ atoms in the ICD process in Kr-rich clusters, whereas in smaller clusters containing less $\mathrm{Kr}$ the surface component seems to play a more important role.

\section{Acknowledgements}

UH would like to acknowledge valuable discussion with Vitali Averbukh and Markus Drescher. The authors would like to thank the Deutsche Forschungsgemeinschaft (DFG), the Advanced Study Group of the Max-Planck-Society and the Fonds der Chemischen Industrie for financial support. The BESSY II staff is also acknowledged for the support during the measurements. 


\section{References}

${ }^{1}$ P. Auger, J. Phys. Radium 6, 205 (1925).

${ }^{2}$ L. S. Cederbaum, J. Zobeley and F. Tarantelli, Phys. Rev. Lett. 79, 4778 (1997).

${ }^{3}$ S. Marburger, O. Kugeler, U. Hergenhahn and T. Möller, Phys. Rev. Lett. 90, 203401 (2003).

${ }^{4}$ T. Jahnke, A. Czasch, M.S.Schöffler, S.Schössler, A.Knapp, M.Käsz, J.Titze, C.Wimmer, K.Kreidi, R.E.Grisenti, A.Staudte, O.Jagutzki, U.Hergenhahn, H.Schmidt-Böcking, and R.Dörner, Phys. Rev. Lett. 93, 163401 (2004).

${ }^{5}$ M. Mucke, M. Braune, S. Barth, M. Förstel, T. Lischke, V. Ulrich, T. Arion, U. Becker, A. Bradshaw and U. Hergenhahn, Nature Phys. 6, 143 (2010).

${ }^{6}$ T. Jahnke, H. Sann, T. Havermeier, K. Kreidi, C. Stuck, M. Meckel, M. Schöffler, N. Neumann, R. Wallauer, S. Voss, A. Czasch, O. Jagutzki, A. Malakzadeh, F. Afaneh, T. Weber, H. Schmidt-Böcking, and R. Dörner, Nature Phys. 6, 139 (2010).

${ }^{7}$ V. Averbukh, P. V. Demekhin, P. Kolorenc, S. Scheit, S. D. Stoychev, A. I. Kuleff, Y.-C. Chiang, K. Gokhberg, S. Kopelke, N. Sisourat, and L. S. Cederbaum, J. Electron Spectrosc. Relat. Phenom. doi: 10.1016/j.elspec.2010.03.003

${ }^{8}$ U. Hergenhahn, J. Electron Spectrosc. Relat. Phenom. doi: 10.1016/j.elspec.2010.12.020

${ }^{9}$ M. Förstel, M. Mucke, T. Arion, A. M. Bradshaw and U. Hergenhahn, Phys. Rev. Lett. 106, $033402(2011)$.

${ }^{10}$ K. Sakai, S. Stoychev, T. Ouchi, I. Higuchi, M. Schöffler, T. Mazza, H. Fuzukawa, K. Nagaya, M. Yao, Y. Tamenori, A. I. Kuleff, N. Saito and K. Ueda, Phys. Rev. Lett. 106, $033401(2011)$. 
${ }^{11}$ J. Zobeley, L. S. Cederbaum and F. Tarantelli, J. Chem. Phys. 108, 9737 (1998).

${ }^{12}$ J. Zobeley, L. S. Cederbaum and F. Tarantelli, J. Phys. Chem. A 103, 11145 (1999).

${ }^{13}$ R. Santra, J. Zobeley, and L. S. Cederbaum, Phys. Rev. B 64, 245104 (2001).

${ }^{14}$ I. B. Müller and L. S. Cederbaum, J. Chem. Phys. 122, 094305 (2005).

${ }^{15}$ V. Averbukh and L. S. Cederbaum, J. Chem. Phys. 123, 204107 (2005).

${ }^{16}$ G. Öhrwall, M. Tchaplyguine, M. Lundwall, R. Feifel, H. Bergersen, T. Rander, A.

Lindblad, J. Schulz, S. Peredkov, S. Barth, S. Marburger, U. Hergenhahn, S. Svensson, and O.

Björneholm, Phys. Rev. Lett. 93, 173401 (2004).

${ }^{17}$ J. Farges, M. F. de Feraudy, B. Raoult and G. Torchet, J. Chem. Phys. 84, 3491 (1986).

${ }^{18}$ M. Förstel, M. Mucke, T. Arion, T. Lischke, S. Barth, V. Ulrich, G. Öhrwall, O.

Björneholm, U. Hergenhahn, and A. M. Bradshaw, Phys. Rev. B 82, 125450 (2010).

${ }^{19}$ M. Förstel, M. Mucke, T. Arion, T. Lischke, S. Barth, V. Ulrich, G. Öhrwall, O.

Björneholm, U. Hergenhahn, and A. M. Bradshaw, J. Electron Spectrosc. Relat. Phenom., http://dx.doi.org/10.1016/j.elspec.2010.09.001

${ }^{20}$ S. Barth, S. Marburger, S. Joshi, V. Ulrich, O. Kugeler and U. Hergenhahn, Phys. Chem. Chem. Phys. 8, 3218 (2006).

${ }^{21}$ M. Lundwall, W. Pokapanich, H. Bergersen, A. Lindblad, T. Rander, G. Öhrwall, M. Tchaplyguine, S. Barth, U. Hergenhahn, S. Svensson, and O. Björneholm, J. Chem. Phys. 126, 214706 (2007).

${ }^{22}$ N. Sisourat, H. Sann, N. V. Kryzhevoi, P. Kolorenč, T. Havermeier, F. Sturm, T. Jahnke, H.-K. Kim, R. Dörner, and L. S. Cederbaum, Phys. Rev. Lett. 105, 173401 (2010). 
${ }^{23}$ K. Ueda, H. Fukuzawa, X.-J. Liu, K. Sakai,G.Prümper, Y. Morishita, N. Saito, I.H. Suzuki, K. Nagaya, H. Iwayama, M. Yao, K. Kreidi, M. Schöffler, T. Jahnke, S. Schössler, R. Dörner, Th.Weber, J. Harries, Y. Tamenori, J. Electron Spectrosc. Relat. Phenom. 166-167, 3 (2008).

${ }^{24}$ S. Scheit, V. Averbukh, H.-D. Meyer, J. Zobeley and L. S. Cederbaum, J. Chem. Phys. 124,154305 (2006).

${ }^{25}$ A. I. Kuleff and L. S. Cederbaum, Phys. Rev. Lett. 98, 083201 (2007).

${ }^{26}$ P. V. Demekhin, Y.-C. Chiang, S. D. Stoychev, P. Kolorenč, S. Scheit, A. I. Kuleff, F. Tarantelli, and L. S. Cederbaum, J. Chem. Phys. 131, 104303 (2009).

${ }^{27}$ V. Averbukh, I. B. Müller, and L. S. Cederbaum, Phys. Rev. Lett. 93, 263002 (2004).

${ }^{28}$ N. V. Kryzhevoi, V. Averbukh, and L. S. Cederbaum, Phys. Rev. B 76, 094513 (2007).

${ }^{29}$ N. Sisourat, N. V. Kryzhevoi, P. Kolorenč, S. Scheit, T. Jahnke, and L. S. Cederbaum, Nature Phys. 6, 508 (2010).

${ }^{30}$ T. Havermeier, T. Jahnke, K. Kreidi, R. Wallauer, S. Voss, M. Schöffler, S. Schössler, L. Foucar, N. Neumann, J. Titze, H. Sann, M. Kühnel, J. Voigtsberger, A. Malakzadeh, N. Sisourat, W. Schöllkopf, H. Schmidt-Böcking, R. E. Grisenti, and R. Dörner, Phys. Rev. Lett. 104, 153401 (2010).

31 A. Lindblad, H. Bergersen, T. Rander, M. Lundwall, G. Öhrwall, M. Tchaplyguine, S. Svensson and O. Björneholm, Phys. Chem. Chem. Phys. 8, 1899 (2006).

${ }^{32}$ D. R. Miller, in Atomic and Molecular Beam Methods Vol. 1, edited by G. Scoles (Oxford University Press, Oxford, 1986).

${ }^{33}$ A. Kanaev, L. Museur, F. Edery, T. Laarmann, and T. Möller, Phys. Rev. B 69, 125343 (2004). 
${ }^{34}$ J. F. Ogilvie and F. Y. H. Wang, J. Mol. Struct. 273, 277 (1992).

35 J. F. Ogilvie and F. Y. H. Wang, J. Mol. Struct. 291, 313 (1993).

${ }^{36}$ D. C. Patton and M. R. Pederson, Int. J. Quant. Chem. 69, 619 (1998).

${ }^{37}$ S. Marburger, PhD Thesis, Technical University Berlin (2004).

${ }^{38}$ S. P. Marburger, O. Kugeler, and U. Hergenhahn, in Synchrotron Radiation

Instrumentation: Eighth International Conference, edited by T. Warwick, J. Arthur, H. A.

Padmore and J. Stöhr (American Institute of Physics, San Francisco, 2003), AIP Conference Proceedings Vol. 705, p. 1114.

${ }^{39}$ See supplementary material at ... for a table of the expansion conditions relevant for the data shown here.

${ }^{40}$ P. Kruit and F. H. Read, J. Phys. E: Sci. Instrum. 16, 313 (1983).

${ }^{41}$ P. Lablanquie, L. Andric, J. Palaudoux, U. Becker, M. Braune, J. Viefhaus, J. H. D. Eland, and F. Penent, J. Electron Spectrosc. Relat. Phenom. 156-158, 51 (2007).

${ }^{42}$ M. Mucke, T. Lischke, T. Arion, M. Förstel, A. M. Bradshaw and U. Hergenhahn, unpublished.

${ }^{43}$ M. Förstel, T. Arion, M. Mucke, A. M. Bradshaw, U. Hergenhahn, C. Domesle, B. J. Jordon-Thaden, A. Wolf, H. B. Pedersen and L. Lammich, unpublished.

${ }^{44}$ C. Domesle, B. Jordon-Thaden, L. Lammich, M. Förstel, U. Hergenhahn, A. Wolf, and H.B.Pedersen, Phys. Rev. A 82, 033402 (2010).

${ }^{45}$ N. Moiseyev, R. Santra, J. Zobeley and L. S. Cederbaum, J. Chem. Phys. 114, 7351 (2001). 
${ }^{46}$ S. Scheit, V. Averbukh, H. D. Meyer, N. Moiseyev, R. Santra, T. Sommerfeld, J. Zobeley and L. S. Cederbaum, J. Chem. Phys. 121, 8393 (2004).

${ }^{47}$ S. Scheit, H. D. Meyer and L. S. Cederbaum, J. Phys.: Conf. Series. 4, 277 (2005).

${ }^{48}$ W. Persson, Physica Scripta 3, 133 (1971).

${ }^{49}$ K. Harth, M. Raab, and H. Hotop, Z. Phys. D 7, 213 (1987).

${ }^{50}$ E. B. Saloman, J. Phys. Chem. Ref. Data 36, 215 (2007).

${ }^{51}$ T. P. Haley and S. M. Cybulski, J. Chem. Phys. 119, 5487 (2003).

${ }^{52}$ R. Feifel, M. Tchaplyguine, G. Öhrwall, M. Salonen, M. Lundwall, R. R. T. Marinho, M.

Gisselbrecht, S. L. Sorensen, A. Naves de Brito, L. Karlsson, N. Mårtensson, S. Svensson, and O. Björneholm, Eur. Phys. J. D 30, 343 (2004).

${ }^{53}$ L. O. Werme, T. Bergmark and K. Siegbahn, Physica Scripta 6, 141, (1972).

${ }^{54}$ S. Peredkov, A. Kivimäki, S. L. Sorensen, J. Schulz, N. Mårtensson, G. Öhrwall, M. Lundwall, T. Rander, A. Lindblad, H. Bergersen, S. Svensson, O. Björneholm, and M. Tchaplyguine, Phys. Rev. A 72, 021201R (2005).

${ }^{55}$ L. Avaldi, G. Dawber, N. Gulley, H. Rojas, G. C. King, R. Hall, M. Stuhec, and M. Zitnik, J. Phys. B 30, 5197 (1997).

${ }^{56}$ L. S. Cederbaum, (2010) private communication.

${ }^{57}$ M. Lundwall, H. Bergersen, A. Lindblad, G. Öhrwall, M. Tchaplyguine, S. Svensson and O. Björneholm, Phys. Rev. A 74, 043206, (2006). 


\section{Figure captions}

Figure 1. Center (panel a)): Intensity of electron-electron coincidences detected from $\mathrm{NeKr}$ mixed clusters recorded at the photon energy $\mathrm{h} v=110 \mathrm{eV}$ shown as a color-coded map (linear color scale). The initial gas mixture contained $5 \% \mathrm{Kr}$. Right-hand side (panel c)) and upperleft (panel b)) panels: electron energy spectra as obtained by integration along the $\mathrm{e}_{1}$ and $\mathrm{e}_{2}$ energy axes, respectively (see text for details).

Figure 2. Center: Photoelectron-ICD electron coincidence spectrum of $\mathrm{NeKr}$ mixed clusters (3\% $\mathrm{Kr}$ in $\mathrm{NeKr}$ mixture), after ionization by $\mathrm{h} v=55 \mathrm{eV}$ shown as a color-coded map (linear color scale). Top-left and bottom-right: The photoelectron and ICD electron spectra of $\mathrm{NeKr}$ mixed clusters, respectively, as obtained from the coincident spectra by projecting the map on the respective axis (see text for details).

Figure 3. ICD spectra of NeKr mixed clusters as recorded at hv $=55 \mathrm{eV}$ (a) and $\mathrm{h} v=110 \mathrm{eV}$ (b) for various levels of $\mathrm{Kr}$ admixture in the coexpansion. Triangles: $2 \% \mathrm{Kr}$, circles: $3 \% \mathrm{Kr}$ and squares: $5 \% \mathrm{Kr}$ in $\mathrm{NeKr}$ mixture. The dashed vertical lines indicate the center of gravity of the three ICD peaks. The estimated dimer transition energy is marked by an arrow (see text for details). 
Supplementary material for article:

\section{Interatomic Coulombic Decay in mixed NeKr clusters}

Tiberiu Arion, Melanie Mucke, Marko Förstel, Alex M. Bradshaw, Uwe Hergenhahn

In the following table the exact conditions of the supersonic expansion used to produce $\mathrm{NeKr}$ clusters are given. In all cases a conical copper nozzle, $15^{\circ}$ half opening angle and $100 \mu \mathrm{m}$ smallest diameter, was used.

\begin{tabular}{|c|c|c|c|}
\hline Figure & $\begin{array}{l}\text { Kr fraction in initial } \\
\text { gas mixture }\end{array}$ & $\begin{array}{l}\text { Stagnation pressure } \\
\text { (bar) }\end{array}$ & $\begin{array}{l}\text { Nozzle temperature } \\
\text { (K) }\end{array}$ \\
\hline 1 & $5 \%$ & 2.3 & 112 \\
\hline 2 & $3 \%$ & $1.9-1.5$ & 110 \\
\hline $\mathbf{3 a}$ & $5 \%$ & 2.3-2.2 & $112.5-116$ \\
\hline $3 \mathbf{a}$ & $3 \%$ & 1.7 & 115 \\
\hline $3 \mathbf{a}$ & $2 \%$ & 1.5 & 120 \\
\hline $3 \mathbf{b}$ & & see Fig. 1 & \\
\hline $3 \mathbf{b}$ & $3 \%$ & 1.5 & 110 \\
\hline $3 b$ & $2 \%$ & 1.2 & 119 \\
\hline
\end{tabular}

